\title{
CircCSNKIGI Contributes to the Development of Colorectal Cancer by Increasing the Expression of MYO6 via Competitively Targeting miR-455-3p
}

This article was published in the following Dove Press journal: Cancer Management and Research

\author{
Xianming Huang' \\ Xiaohua Shen ${ }^{2}$ \\ Lixiang Peng ${ }^{2}$ \\ Wenli $\mathrm{Mai}^{2}$ \\ Yiye Wan ${ }^{2}$ \\ Huiqing Zhang ${ }^{2}$ \\ 'Department of Pathology, Jiangxi Cancer \\ Hospital, Nanchang, Jiangxi 330029 , \\ People's Republic of China; ${ }^{2}$ Department \\ of Gastrointestinal Medical Oncology, \\ Jiangxi Cancer Hospital, Nanchang, \\ Jiangxi 330029, People's Republic of \\ China
}

Background: Numerous circular RNAs (circRNAs) are functionally investigated in various human cancers, including colorectal cancer (CRC). In this study, we explored the function of circCSNK1G1 and mechanism of action in CRC, aiming to provide evidence for circCSNK1G1 involving in CRC pathogenesis.

Methods: The expression of circCSNK1G1, miR-455-3p and Myosin VI (MYO6) were examined using quantitative real-time polymerase chain reaction (qRT-PCR). The functions of circCSNK1G1 on cell proliferation, apoptosis, cycle and migration/invasion were investigated using 3-(4, 5-dimethylthiazol-2-yl)-2, 5-diphenyltetrazolium bromide (MTT) assay, colony formation assay, flow cytometry assay and transwell assay, respectively. The targeted relationship between miR-455-3p and circCSNK1G1 or MYO6 predicted by bioinformatics analysis was validated using dual-luciferase reporter assay and RNA pull-down assay. The role of circCSNK1G1 was also explored in nude mice in vivo.

Results: The expression of circCSNK1G1 and MYO6 was elevated, while the expression of miR-455-3p was declined in CRC tissues and cells. Silencing circCSNK1G1 inhibited CRC cell proliferation, migration and invasion and induced cell apoptosis and cell cycle arrest. MiR-455-3p was a target of circCSNK1G1, and miR-455-3p could bind to MYO6. CircCSNK1G1 positively regulated MYO6 expression by targeting miR-455-3p. Inhibition of miR-455-3p reversed the effects of circCSNK1G1 silencing in CRC cells. Besides, miR455-3p restoration blocked CRC cell growth and metastasis, which were abolished by MYO6 overexpression. Moreover, circCSNK1G1 regulated the miR-455-3p/MYO6 axis to block tumor growth in vivo.

Conclusion: CircCSNK1G1 participated in the progression of CRC partly by modulating the miR-455-3p/MYO6 network, which provided a theoretical basis for circCSNK1G1 involving in CRC pathogenesis, hinting that circCSNK1G1 might be a useful biomarker for CRC treatment.

Keywords: circCSNK1G1, miR-455-3p, MYO6, colorectal cancer

\section{Introduction}

Colorectal cancer (CRC) is one of the most common cancers worldwide. ${ }^{1}$ About one to two million new cases are diagnosed each year, making CRC the third most common type of cancer. ${ }^{1}$ In terms of the gender of the case, CRC is the second most common cancer in women and the third most common cancer in men. ${ }^{2}$ Adenocarcinoma occupies the majority of CRC according to classification. ${ }^{3}$ The risk of CRC is related to personal characteristics or habits, such as age, chronic medical history, and lifestyle. ${ }^{4-6}$ In addition, studies have shown that abnormal
Correspondence: Huiqing Zhang

Tel +8679188313831

Email huiqingzhang@outlook.com 
changes in non-coding RNAs (ncRNAs), such as long ncRNA (IncRNA), circular RNA (circRNA) or microRNA (miRNA), may also affect cancer progression and have predictive value. ${ }^{1,7}$ Therefore, the management and development of different ncRNAs should be valued in CRC to improve prognosis and treatment options.

CircRNA is a novel type of ncRNA, well known for its single-stranded, ring-closed structure. ${ }^{8}$ CircRNAs are generated from pre-mRNAs in a "backsplicing" mechanism. 9 Compared to linear mRNA molecules, however, circRNAs are stably expressed and widely distributed in most subcellular compartments of eukaryotic cells. ${ }^{8,10}$ Hence, circRNAs are deemed as more promising biomarkers for various human diseases. ${ }^{11}$ Now, much attention of circRNAs is attracted because of their contribution to cancer initiation and development. Dysregulation of circRNAs is involved in multiple malignant activities of cancer cells, such as aberrant proliferation, epithelialmesenchymal transition, migration, invasion and metabolism. ${ }^{12-14}$ Due to the development of sequencing technology, a growing number of differently expressed circRNAs in tumor tissues and normal tissues have been screened and identified. ${ }^{15}$ CircCSNK1G1 is generated from linear CSNK1G1 and identified to be aberrantly expressed in CRC tissues. ${ }^{16}$ However, its detailed role in $\mathrm{CRC}$ is unclear.

MiRNAs are mature and widely studied biomarkers in cancers, including CRC. ${ }^{17}$ MiRNAs-induced deregulation has been well recorded and continues to develop in CRC, such as miR-760, miR-1258 and miR-143. ${ }^{18-20}$ Interestingly, miR-455-3p was also a vital regulator in $\mathrm{CRC}^{21}$ and the associated functional mechanism of miR455-3p in CRC should be enriched. Myosin VI (MYO6) is a member of myosin proteins, actin-based motor proteins. $^{22}$ MYO6 plays essential functions in intracellular transportation, such as vesicular membrane traffic, cytokinesis and migration. ${ }^{23}$ MYO6 is further explored to be associated with cancer cell growth and metastasis, thereby affecting cancer progression. ${ }^{22,24}$ The role of MYO6 in $\mathrm{CRC}$ is insufficient, and its mechanism linked to circCSNK1G1 or miR-455-3p has not been mentioned.

Currently, we screened circCSNK1G1 from Gene Expression Omnibus (GEO) datasets and validated the expression of circCSNK1G1, miR-455-3p and MYO6 in clinical CRC tissues. Gain- or loss-function experiments were performed to ascertain the function of circCSNK1G1 in vitro and in vivo. Our objective was to explore the biological functions of circCSNK1G1 in CRC and provide a regulatory mechanism of circCSNK1G1.

\section{Materials and Methods CircRNA Expression from GEO}

CircRNA expression profile was downloaded from GEO (accession: GSE126094; https://www.ncbi.nlm.nih.gov/ geo/query/acc.cgi?acc=GSE126094). The circRNA microarray was conducted using $10 \mathrm{CRC}$ tissues and matched normal-appearing tissues. The GEO2R tool (https://www. ncbi.nlm.nih.gov/geo/geo2r) was used to identify differently expressed circRNAs between CRC tissues and normal tissues. Values of $\mid \log 2$ fold change $\mid(|\log 2 \mathrm{FC}|)>1$ and $p<0.05$ were set as cut-off criteria.

\section{Clinical Tissues}

CRC tumor tissues $(n=55)$ and paired normal tissues were collected from CRC patients who were diagnosed and surgically removed at Jiangxi Cancer Hospital. Each patient had signed the written informed consent. These excised tissues were quick-frozen in liquid nitrogen and placed in $-80^{\circ} \mathrm{C}$ freezer. The Ethics Committee of Jiangxi Cancer Hospital approved this research (No.2019JX776). The research has been carried out in accordance with the World Medical Association Declaration of Helsinki.

\section{Cell Lines}

CRC cell lines, including HCT116 and SW620 cells, and normal colorectal epithelial cells (NCM460) were purchased from Procell Co., Ltd. (Wuhan, China) and maintained in McCoy's medium (Procell Co., Ltd.) containing 10 fetal bovine serum (FBS), Leibovitz's L-15 medium (Procell Co., Ltd.) containing 10\% FBS and M199 medium (Procell Co., Ltd.) containing 10\% FBS, respectively. Cells cultured in the corresponding culture medium were placed in an incubator containing $5 \% \mathrm{CO}_{2}$ at $37^{\circ} \mathrm{C}$.

\section{Quantitative Real-Time Polymerase Chain Reaction (qRT-PCR)}

Total RNA was isolated using a commercial Trizol reagent (KeyGen Biotech, Nanjing, China) following the procedures. Subsequently, complementary DNA (cDNA) was synthesized using the cDNA Reverse Transcription Kits (Thermo Fisher Scientific, Waltham, MA, USA) or MicroRNA Reverse Transcription Kit (Thermo Fisher Scientific). Then, cDNA was amplified by qRT-PCR using the SYBR Green Mix (Thermo Fisher Scientific) 
under a CFX96 PCR system (Bio-Rad, Hercules, CA, USA) with glyceraldehyde-3-phosphate dehydrogenase (GAPDH) or U6 as house-keeping gene. The final relative expression was expressed using the $2^{-\Delta \Delta \mathrm{Ct}}$ method. ${ }^{25}$ All primer sequences were listed as below:

CircCSNK1G1: 5'-GGTGCATCTGCAATAACTCG-3'

(F) and 5'-ATTTCCCACATGGTCCAAAG-3' (R); CSNK1G1: 5'- TGTATCATCAGAGCGCCGAG-3' (F) and 5'-TGAGCTAACCACCTGCACTG-3' (R); GAPDH: 5'-CTTTGGTATCGTGGAAGGACTC-3' (F) and 5'TCTTCCTCTTGTGCTCTTGCTG-3' (R); miR-455-3p: 5'- TAAGACGTCCATGGGCAT-3' (F) and 5'-GTGCA GGGTCCGAGGT-3' (R); U6: 5'-AGAGCCTGTGGTGT CCG-3' (F) and 5'-CATCTTCAAAGCACTTCCCT-3' (R); MYO6: 5'-GATGGAGCTGCACCCTGACA-3' (F) and 5'GCTCTCAATGGCGCTCTGAAG-3' (R).

\section{Actinomycin D (ActD) Treatment}

HCT116 and SW620 cells were exposed to ActD (50 ng/ mL; Cell Signaling Technology, Danvers, MA, USA) for 4, 8, 12 and $24 \mathrm{~h}$. Cells were then harvested and used for RNA isolation. Next, qRT-PCR was carried out to detect the expression of circCSNK1G1 and CSNK1G1.

\section{RNase R Digestion}

The isolated RNAs were exposed to RNase R (2 U/ $\mu \mathrm{g}$ RNA, Epicentre, Madison, WI, USA) for 15 minutes at $37^{\circ} \mathrm{C}$, followed by the qRT-PCR analysis to detect the expression of circCSNK1G1 and CSNK1G1. Untreated RNAs were used as the control (Mock).

\section{Subcellular Distribution}

Nuclear RNA and cytoplasmic RNA were extracted using the Cytoplasmic \& Nuclear RNA purification kit (Norgen Biotek, Thorold, Canada) and used for qRT-PCR analysis to detect the expression of circCSNK1G1. GAPDH or U6 was used as the control of cytoplasmic fraction and nuclear fraction, respectively.

\section{Cell Transfection}

For circCSNK1G1 knockdown, small interference RNA (siRNA) targeting circCSNK1G1 (si-circCSNK1G1) was synthesized by Genepharma (Shanghai, China) with si-NC as the negative control. For miR-455-3p enrichment or inhibition, miR-455-3p mimics (miR-455-3p) or miR-455-3p inhibitors (anti-miR-455-3p) were purchased from Ribobio (Guangzhou, China) with miR-NC or anti-miR-NC as the negative control. For MYO6 overexpression, MYO6 sequence was amplified and cloned into pcDNA plasmid (Genepharma), named as MYO6, with empty vector as the control. Cell transfection was conducted in CRC cells using Lipofectamine 3000 (Invitrogen, Carlsbad, CA, USA).

\section{3-(4, 5-Dimethylthiazol-2-YI)-2, 5-Diphenyltetrazolium Bromide (MTT) Assay}

HCT116 and SW620 cells planted into 96-well plates (2000 cells/well) were subjected to diverse transfection. MTT Cell Proliferation Assay Kit (Sangon Biotech, Shanghai, China) was utilized to detect cell proliferation according to the protocol at different times (24, 48 and 72 h). The absorbance was measured at $490 \mathrm{~nm}$ under a microplate reader (Thermo Fisher Scientific).

\section{Colony Formation Assay}

To further observe cell proliferation, colony formation assay was arranged. In brief, HCT116 and SW620 cells with diverse transfection were seeded into 6-well plates (1000 cells/well) and cultured at $37^{\circ} \mathrm{C}$ incubator for 12 days. Then, colonies were fixed with methanol and stained with $0.1 \%$ crystal violet followed by the investigation under a light microscope (Olympus, Tokyo, Japan).

\section{Flow Cytometry Assay}

Cell apoptosis was investigated using Annexin V Apoptosis Detection Kit (Sangon Biotech). In brief, HCT116 and SW620 cells with different transfections were resuspended in $195 \mu \mathrm{L}$ binding buffer $\left(4 \times 10^{5}\right.$ cells). Then, cells were exposed to Annexin V-fluorescein isothiocyanate (FITC) for $15 \mathrm{~min}$ followed by the treatment of $10 \mu \mathrm{L}$ propidium iodide (PI). Subsequently, the apoptotic cells were distinguished by flow cytometry (BD Bioscience, SanJose, CA, USA).

Cell cycle was monitored using Cell Cycle Assay Kit for Flow cytometry (Sangon Biotech). In brief, cells were collected and resuspended in phosphate-buffered saline (PBS). Then, cells were fixed in $70 \%$ pre-cooled alcohol and incubated at $-20^{\circ} \mathrm{C}$ overnight followed by the treatment of RNase A. Then, cells were stained with $0.5 \mathrm{~mL}$ PI at $4{ }^{\circ} \mathrm{C}$ in the dark. Cell cycle distribution was analyzed using flow cytometry (BD Bioscience).

\section{Western Blot}

Total proteins were extracted and electrophoresed by $12 \%$ sodium dodecyl sulfate-polyacrylamide gel electrophoresis 
(SDS-PAGE) followed by the loading on polyvinylidene difluoride membranes (Bio-Rad, Hercules, CA, USA). After blocking in skim milk, the membranes were exposed to the primary antibodies, including anti-B-cell lymphoma-2 (anti-Bcl-2; ab32124; Abcam, Cambridge, MA, USA), anti-Bcl-2-associated X (anti-Bax; ab32503; Abcam, Cambridge, MA, USA), anti-cleaved caspase-3 (anti-c-caspase-3; ab32042; Abcam), anti-MYO6 (ab111694; Abcam) and anti-GAPDH (ab9485; Abcam). After $12 \mathrm{~h}$, the membranes were exposed to the goat antirabbit secondary antibody (ab205718; Abcam) for 1.5 h. Finally, the protein bands were displayed using enhanced chemiluminescence (Sangon Biotech).

\section{Transwell Assay}

Transwell chambers (BD Bioscience) were coated with or without Matrigel (BD Bioscience) at $37^{\circ} \mathrm{C}$ overnight. HCT116 and SW620 cells with diverse transfection for $24 \mathrm{~h}$ were then resuspended in fresh culture medium containing $10 \%$ FBS. The top of chambers without Matrigel was added with resuspended cells for migration assay. The top of chambers with Matrigel was added with resuspended cells for invasion assay. Meantime, the bottom of the chambers was supplemented with fresh culture medium containing $10 \%$ FBS to allow migration or invasion for $24 \mathrm{~h}$. Then, cells in the lower surface were fixed with methanol and subjected to $0.1 \%$ crystal violet. The phenotype was observed under a light microscope (Olympus) with the magnification of $100 \times$.

\section{Dual-Luciferase Reporter Assay}

Bioinformatics analysis was performed to predict the interaction between miR-455-3p and circCSNK1G1 or MYO6 and analyze the binding sites between them using the online database starbase (http://starbase.sysu. edu.cn/). According to the predicted wild-type sequence of binding sites, the sequence of circCSNK1G1 or MYO6 mutated at the binding sites was designed. Then, the wild-type and mutant-type sequences of circCSNK1G1 or MYO6 3'-UTR were amplified and cloned into PGL4 reporter plasmid (Promega, Madison, WI, USA), namely circCSNK1G1 WT, circCSNK1G1 MUT, MYO6 3'-UTR-WT or MYO6 3'-UTR-MUT, respectively. Subsequently, the alone fusion plasmid and $\mathrm{miR}-455-3 \mathrm{p}$ or miR-NC were cotransfected into HCT116 and SW620 cells. After $48 \mathrm{~h}$, the luciferase activity was detected using the Dual-Luciferase Assay System (Promega).

\section{RNA Pull-Down Assay}

MiR-455-3p and miR-NC were labeled using biotin by Ribobio, named as biotin-miR-455-3p and biotin-NC. HCT116 and SW620 cells were transfected with biotinmiR-455-3p or biotin-NC and incubated for 48 h. Afterwards, cells were subjected to the lysis buffer. Subsequent pull-down assay was performed using the Pierce Magnetic RNA Protein Pull-down Kit (Thermo Fisher Scientific). The abundance of circCSNK1G1 was examined by qRT-PCR analysis.

\section{Xenograft Model}

The approval of the Animal Care and Use Committee of Jiangxi Cancer Hospital was obtained before research. Animal studies were performed in compliance with the ARRIVE guidelines and the Basel Declaration. All animals received humane care according to the National Institutes of Health (USA) guidelines. Nude mice $(\mathrm{n}=14, \mathrm{BALB} / \mathrm{C}, 4 \sim 6$ weeks old, male) were purchased from Shanghai Model Organisms (Shanghai, China). Short hairpin RNA (shRNA) targeting circCSNK1G1 (sh-circCSNK1G1) packed into the lentiviral vector was synthesized and constructed by Genepharma, with sh-NC as the negative control. SW620 cells infected with lentivirus containing sh-circCSNK1G1 or sh-NC were implanted into nude mice to establish CRC Xenograft models (shcircCSNK1G1 group and sh-NC group; $\mathrm{n}=7$ per group). After 1-week post-implantation, tumor volume for each mouse was recorded once a week, and mice were sacrificed after 28 days. Tumor tissues were removed to detect tumor weight and expression analysis.

\section{Statistical Analysis}

All experiments were performed in triplicate. Analysis of variance (ANOVA) with Tukey's test was applied to evaluate differences among multiple groups, and Student's $t$-test was applied to evaluate differences between the two groups. Statistical analyses were carried out using GraphPad Prism 7.0 (GraphPad Software, La Jolla, CA, USA), and the data were shown as mean \pm standard deviation. $P$-value less than 0.05 was considered to be statistically significant. 


\section{Result}

CircCSNKIGI Expression Was Enhanced in Tumor Tissues and Cells of CRC, and circCSNKIGI Was Stably Expressed in CRC Cells and Mainly Distributed in the Cytoplasm

Heap map for the 14 most significant differentially expressed circRNAs in CRC tissues $(n=10)$ and paired normal tissues $(n=10)$ was generated from the GEO data (accession: GSE126094), and circCSNK1G1 was significantly upregulated in tumor tissues compared to normal tissues (Figure 1A). Besides, a schematic diagram was depicted to reveal the formation of circCSNK1G1 (circ_0001955), and circCSNK1G1 was derived from exon 5-10 of linear CSNK1G1 mRNA (Figure 1B). In clinical samples, the expression of circCSNK1G1 was also notably elevated in tumor tissues $(n=55)$ compared with that in normal tissues $(n=55)$ (Figure 1C). Likewise, the expression of circCSNK1G1 was visibly promoted in HCT116 and SW620 cells compared with that in NCM460 cells (Figure 1D). Meanwhile, the characteristics of circCSNK1G1 were determined. HCT116 and SW620 cells were exposed to ActD, and the expression of linear CSNK1G1 was strikingly decreased compared to circCSNK1G1 expression (Figure 1E and F). Total RNA isolated from HCT116 and SW620 cells was exposed to RNase R, and the expression of circCSNK1G1 was barely changed, while the expression of linear CSNK1G1 was significantly reduced (Figure $1 \mathrm{G}$ and $\mathrm{H}$ ), showing that circCSNK1G1 was more stable than its parental gene, CSNK1G1. We next examined the subcellular localization of circCSNK1G1, and the data demonstrated that circCSNK1G1 was predominately distributed in the cytoplasm compared with that in the nucleus (Figure 1I and J).

\section{Silencing circCSNKIGI Inhibited Proliferation, Migration and Invasion and Induced Apoptosis and Cell Cycle Arrest in HCTII 6 and SW620 Cells}

Specific circCSNK1G1 siRNA was assembled to knock down the expression of circCSNK1G1 in HCT116 and SW620 cells. The data from qRT-PCR showed that the level of circCSNK1G1 was markedly reduced by sicircCSNK1G1 transfection (Figure 2A and B). Cell proliferation was assessed by MTT assay and colony
A

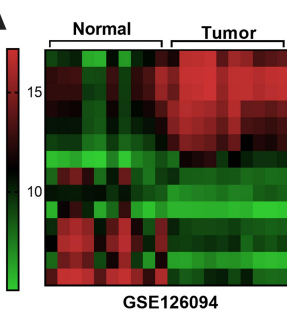

E

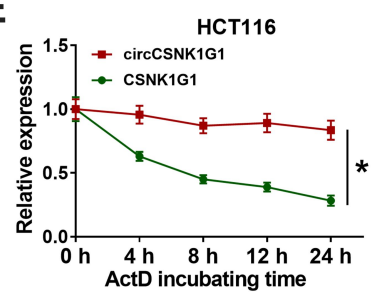

I

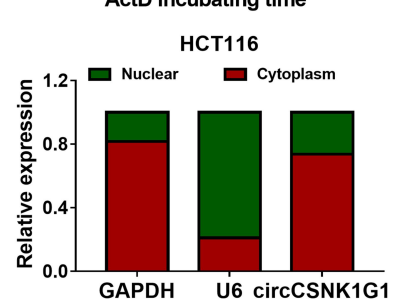

B

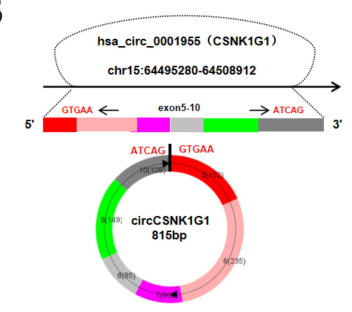

F

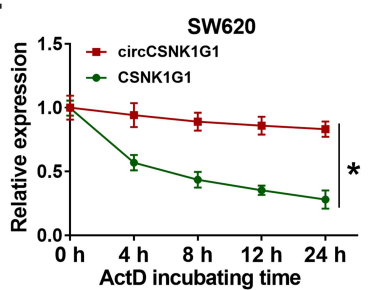

$J$

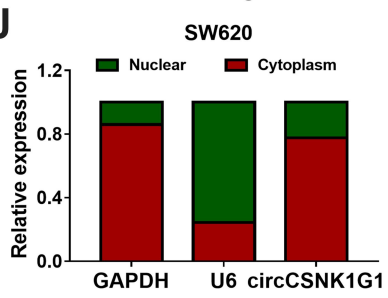

C

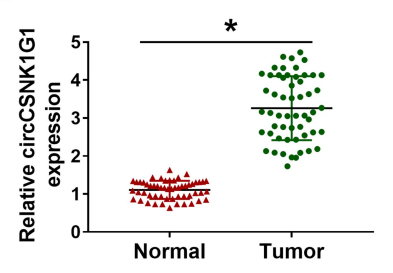

G

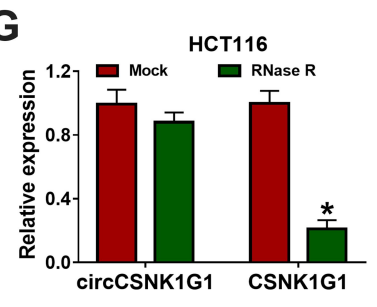

D

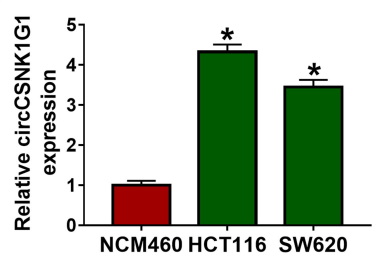

H

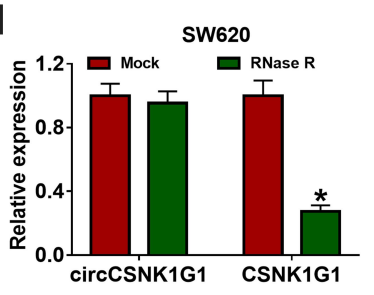

Figure I The expression of circCSNKIGI was reinforced in CRC tissues and cells. (A) Differently expressed circCSNKIGI in CRC tissues was obtained from the GEO dataset. (B) Schematic diagram was depicted to disclose the generation of circCSNKIGI. (C) The expression of circCSNKIGI in CRC tumor tissues ( $\mathrm{n}=55$ ) and normal tissues $(n=55)$ was detected by qRT-PCR. (D) The expression of circCSNKIGI in NCM460, HCTII6 and SW620 cells was detected by qRT-PCR. (E-H) The stability of circCSNKIGI was checked using ActD and RNase R. (I and J) Nuclear and cytoplasmic RNA was isolated to identify the distribution of circCSNKIGI. *P<0.05. 


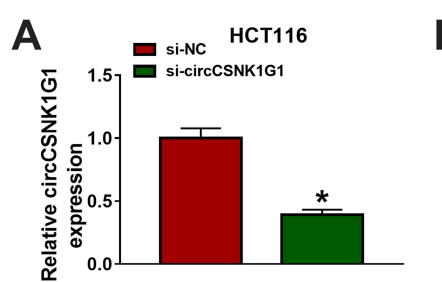

E

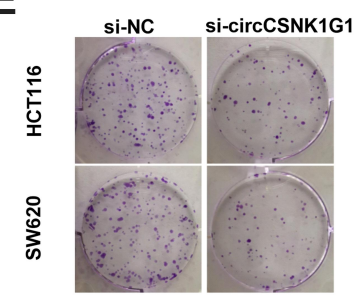

G

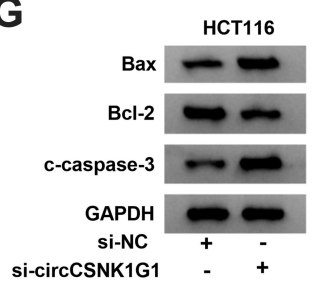

I

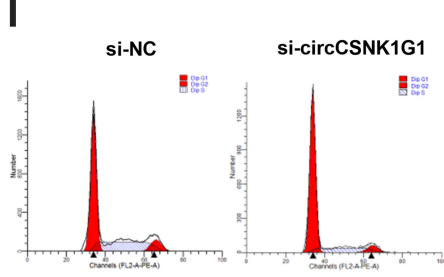

K

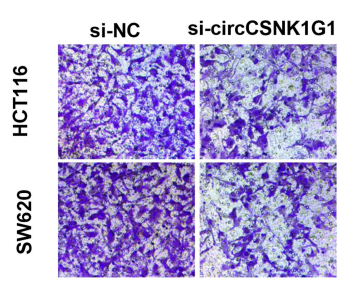

B
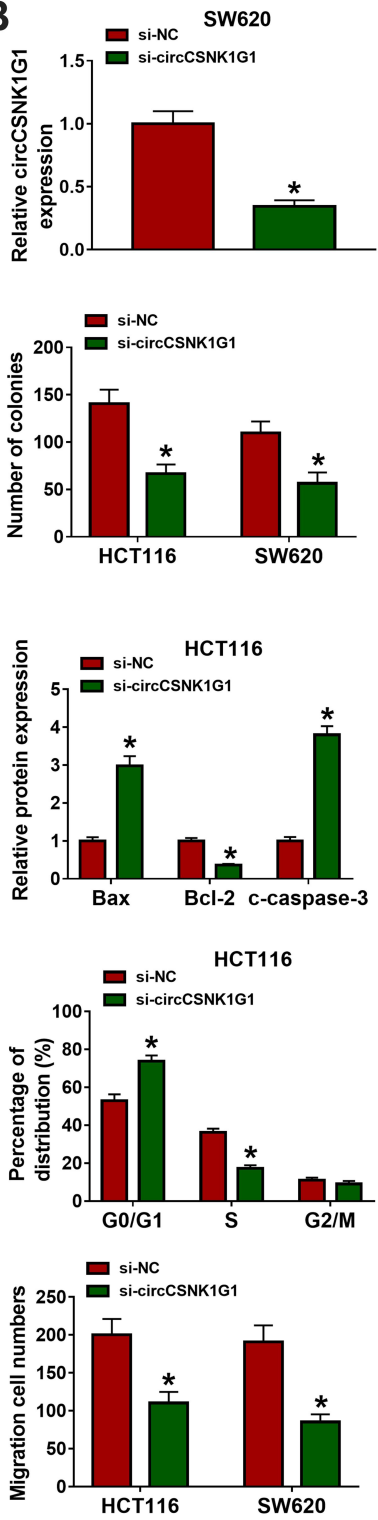

C

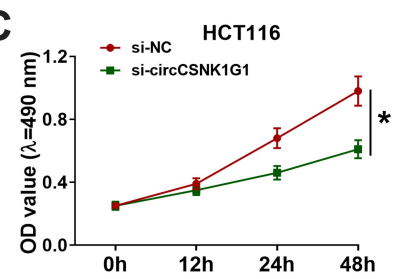

$F$

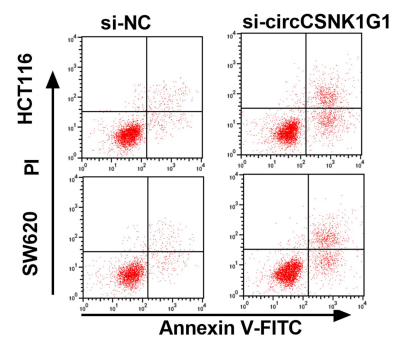

H

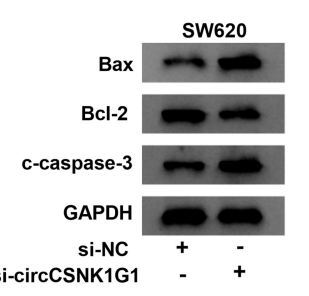

J

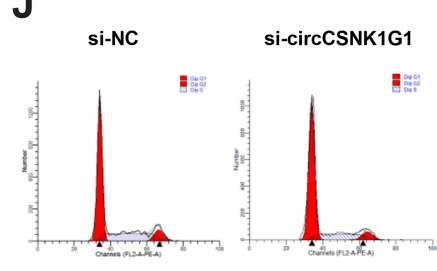

L

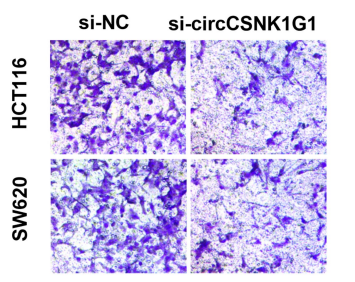

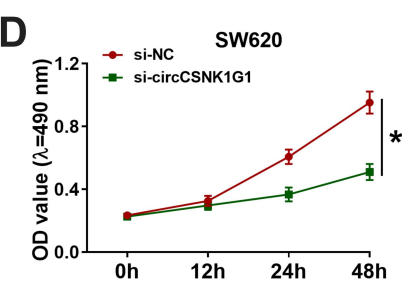
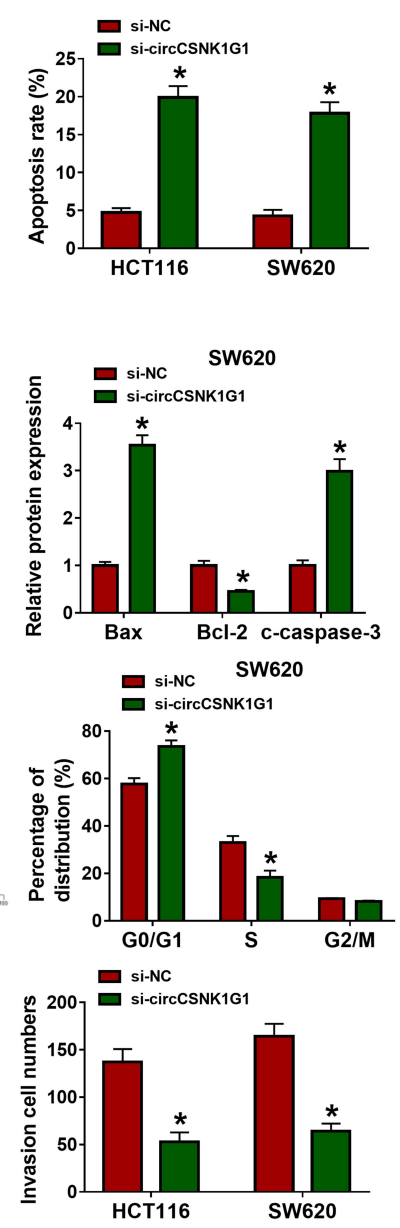

Figure 2 Silencing circCSNKIGI blocked CRC development in vitro. (A and B) The expression of circCSNKIGI in HCTII6 and SW620 cells transfected with sicircCSNKIGI or si-NC was measured by qRT-PCR. (C-E) Cell proliferation was assessed by MTT assay and colony formation assay after circCSNKIGI knockdown. (F) Cell apoptosis was monitored by flow cytometry assay after circCSNKIGI knockdown. (G and $\mathbf{H}$ ) The expression of Bax, Bcl-2 and c-caspase-3 was measured by Western blot after circCSNKIGI knockdown. (I and J) Cell cycle distribution was investigated by flow cytometry after circCSNKIGI knockdown. (K and L) Cell migration and invasion were checked by transwell assay after circCSNKIGI knockdown. $* P<0.05$.

formation assay, and we found that silencing circCSNK1G1 weakened cell OD value and colony number (Figure 2C-E). Cell apoptosis was checked by flow cytometry, and the result suggested that silencing circCSNK1G1 significantly stimulated the number of apoptotic cells (Figure 2F), which was verified by the expression levels of apoptosis-related markers. The expression of Bax and c-caspase-3 was notably increased, while the expression of Bcl-2 was notably decreased in HCT116 and SW620 cells transfected with si-circCSNK1G1 (Figure 2G and $\mathrm{H}$ ). In addition, flow cytometry assay also manifested that circCSNK1G1 silencing induced cell cycle arrest at the G0/G1-S transition (Figure 2I and J). Moreover, transwell assay was performed to detect cell migration and invasion, and the data expressed that the ability of migration and invasion was impaired by circCSNK1G1 silencing (Figure 2K and L). In brief, circCSNK1G1 downregulation suppressed CRC cell growth and metastasis. 


\section{MiR-455-3p Was a Target of circCSNKIG I}

The above experiments showed that circCSNK1G1 was mainly located in the cytoplasm. It was suggested that ncRNAs located in the cytoplasm could sequester target miRNAs and thus blocked miRNA expression and function, terming as sponge effects. ${ }^{26}$ To figure out whether circCSNK1G1 could function as miRNA sponge to play its biological functions, the bioinformatics tool starBase was applied to analyze the potential target miRNAs. As we saw from Figure $3 \mathrm{~A}$, circCSNK1G1 directly targeted miR-455-3p with several binding sites, and we mutated the binding sites of circCSNK1G1 sequence to validate the interaction between circCSNK1G1 and miR-455-3p. Further analysis illustrated that miR-455-3p enrichment significantly diminished the luciferase activities in HCT116 and SW620 cells transfected with circCSNK1G1 WT rather than circCSNK1G1 MUT (Figure 3B and C), and in RNA pull-down assay, biotinmiR-455-3p could significantly enrich the abundance of circCSNK1G1 (Figure 3D). We next ascertained the expression of miR-455-3p in CRC tissues and cells, and we found miR-455-3p was strikingly downregulated in tumor tissues $(n=55)$ and cell lines (HCT116 and SW620) compared with normal tissues $(n=55)$ and cells (NCM460), respectively (Figure $3 \mathrm{E}$ and $\mathrm{F}$ ). Besides, the expression of miR-455-3p was notably strengthened in si-circCSNK1G1-transfected HCT116 and SW620 cells (Figure 3G). It could be sure that miR-455-3p was targeted by circCSNK1G1.

A

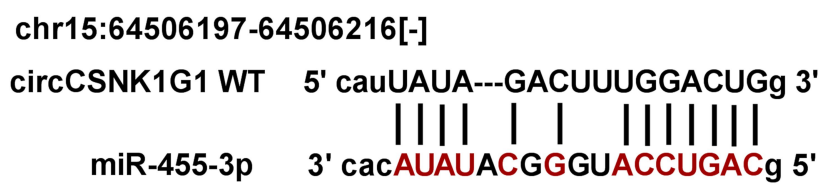

B

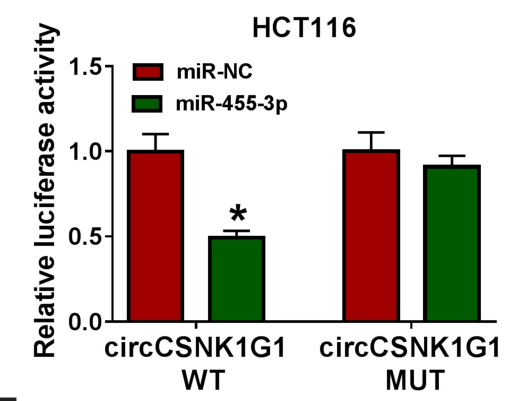

$E$

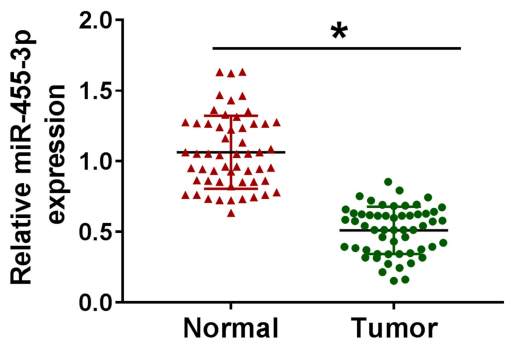

C

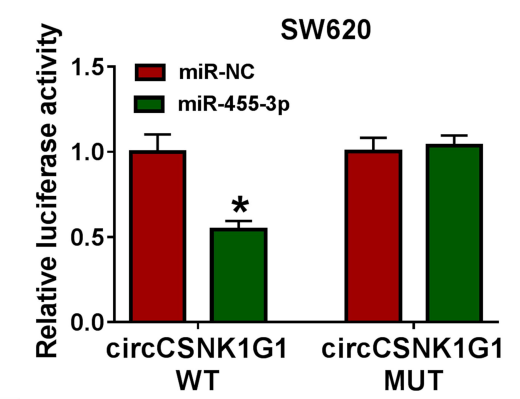

$\mathbf{F}$

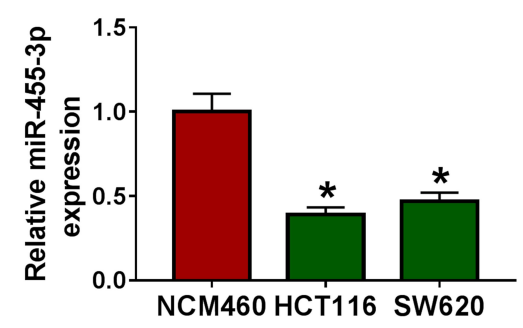

D

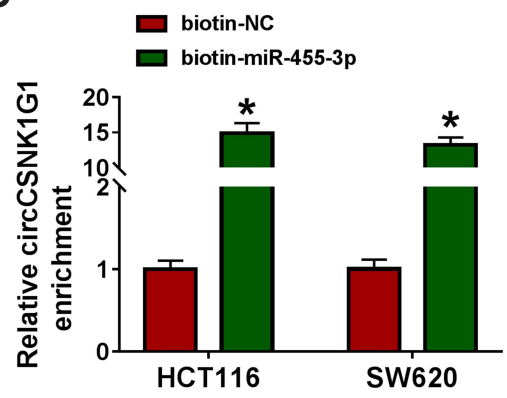

G

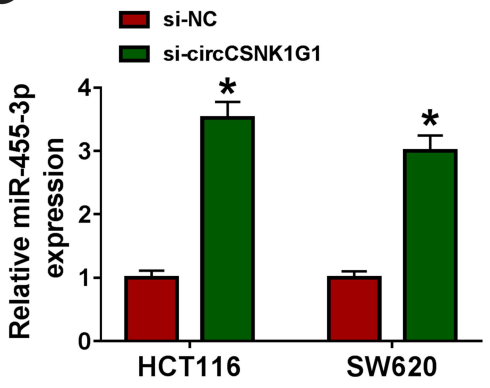

Figure 3 CircCSNKIGI targeted miR-455-3p. (A) Using the bioinformatics tool starBase to predict the relationship between circCSNKIGI and miR-455-3p. (B-D) Using dual-luciferase reporter assay and RNA pull-down assay to validate the interaction between circCSNKIGI and miR-455-3p. (E) The expression of miR-455-3p in clinical tissues was measured by qRT-PCR. (F) The expression of circCSNKIGI in cell lines was measured by qRT-PCR. (G) The expression of circCSNKIGI in HCTII6 and SW620 cells transfected with si-circCSNKIGI was measured by qRT-PCR. $* P<0.05$. 


\section{Inhibition of miR-455-3p Partly Reversed Silencing circCSNKIGI-Blocked Progression of CRC Cells}

We subsequently determined whether circCSNK1G1 regulated CRC progression by modulating miR-455-3p. HCT116 and SW620 cells were suffered from alone circCSNK1G1 silencing or combined circCSNK1G1 silencing and miR-455-3p inhibition. The expression of miR455-3p promoted in cells transfected with sicircCSNK1G1 was notably decreased in cells transfected with si-circCSNK1G1+anti-miR-455-3p (Figure 4A and B). In function, silencing circCSNK1G1-induced inhibition of the value of OD and the number of colonies was abolished by the cotransfection of si-circCSNK1G1 +anti-miR-455-3p, leading to the promotion of cell proliferation (Figure 4C-E). Besides, silencing circCSNK1G1induced cell apoptosis was suppressed by combined circCSNK1G1 silencing and miR-455-3p inhibition (Figure 4F). The expression levels of Bax and c-caspase3 were enhanced in HCT116 and SW620 cells transfected with si-circCSNK1G1 but weakened in cells transfected with si-circCSNK1G1+anti-miR-455-3p, while the expression level of Bcl-2 was opposite to them (Figure 4G and $\mathrm{H})$. In addition, silencing circCSNK1G1-induced cell
A

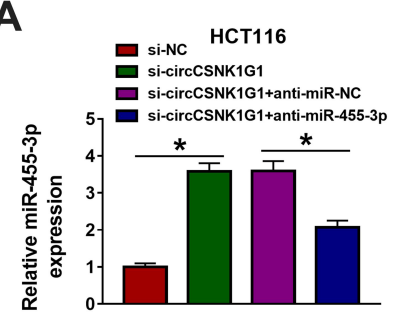

E

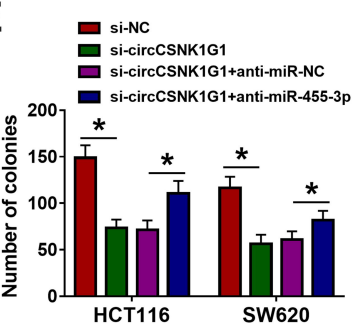

H

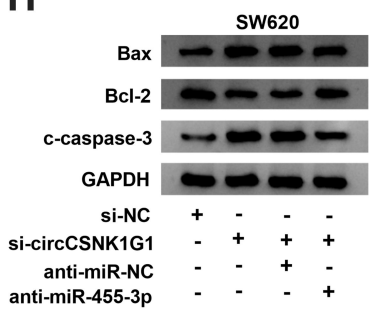

K

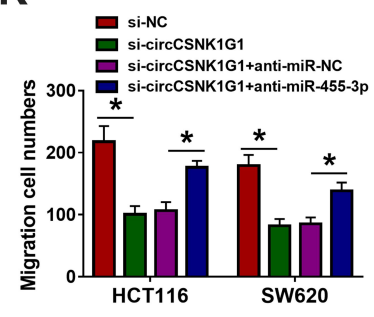

B

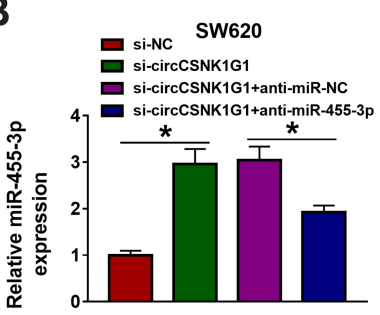

$\mathbf{F}$
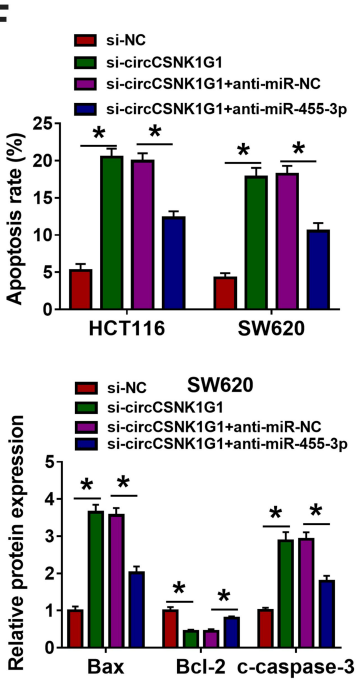

L

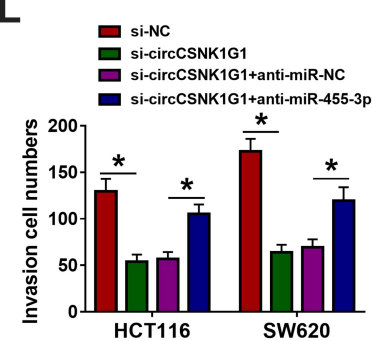

C

$\rightarrow$ si-NC HCT116

$\rightarrow$ si-NC

- si-circCSNK1G1+anti-miR-NC

$\left.\bar{\xi}^{1.2}\right] \rightarrow$ si-circCSNK1G1+anti-miR-455-3p

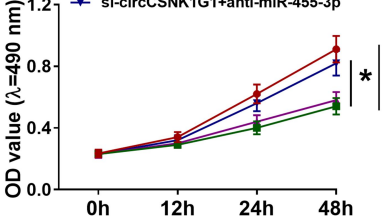

G
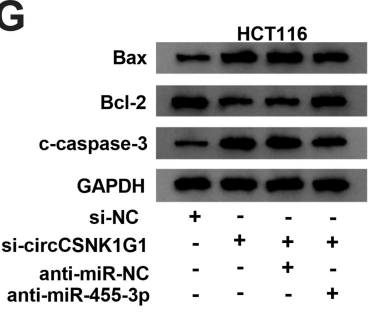

I

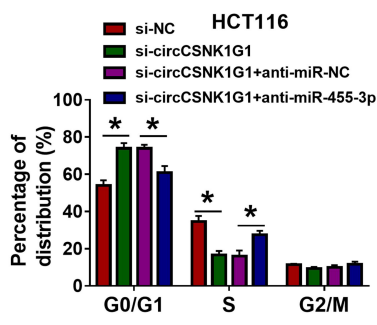

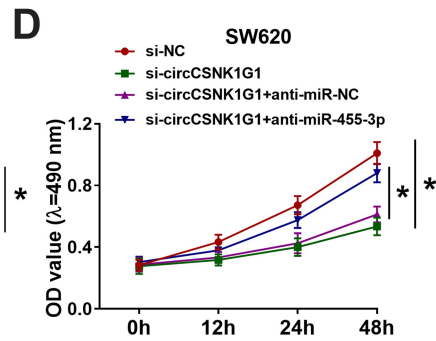

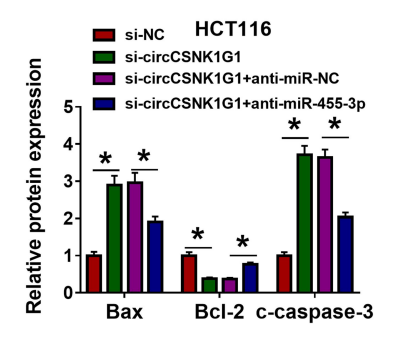

$\mathbf{J}$

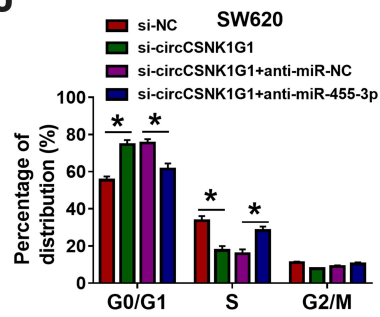

Figure 4 Silencing circCSNKIGI blocked the development of CRC in vitro by increasing miR-455-3p. HCTII6 and SW620 cells were introduced with si-circCSNKIGI, siNC, si-circCSNKIGI+anti-miR-455-3p or si-circCSNKIGI+anti-miR-NC. (A and B) The expression of miR-455-3p in these transfected cells was examined by qRT-PCR. (C-E) Cell proliferation in these transfected cells was monitored by MTT assay and colony formation assay. (F) Cell apoptosis was examined using flow cytometry assay in these transfected cells. ( $\mathbf{G}$ and $\mathbf{H}$ ) The expression of Bax, Bcl-2 and c-caspase-3 was quantified by Western blot in these transfected cells. (I and J) Cell cycle distribution was checked by flow cytometry assay in these transfected cells. ( $\mathbf{K}$ and $\mathbf{L})$ Cell migration and invasion were examined using transwell assay in these transfected cells. ${ }^{*} P<0.05$. 
cycle arrest was relieved by the reintroduction of anti-miR $-455-3 \mathrm{p}$ (Figure 4I and J). Cell migration and invasion blocked in cells with alone si-circCSNK1G1 transfection were activated in cells with combined si-circCSNK1G1 +anti-miR-455-3p transfection (Figure 4K and L). These results strongly suggested that circCSNK1G1 regulated the progression of CRC via targeting miR-455-3p.

A

chr6:76625541-76625547[+]

MYO6 3'-UTR-WT 5' ugGCAUAGUGGCUUAACUGGACUGa 3' miR-455-3p | ||| | || | || || ||| MYO6 3'-UTR-MUT 5' ugACGCGGAGAAUUGACGAUGAGAa 3'

D

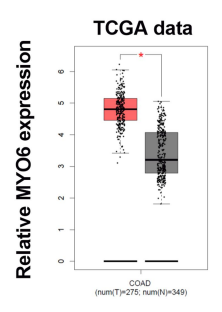

G

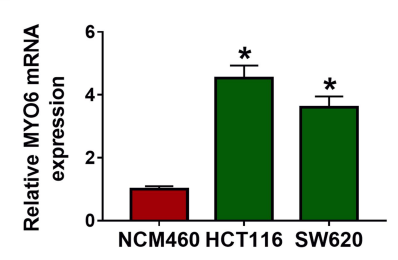

J
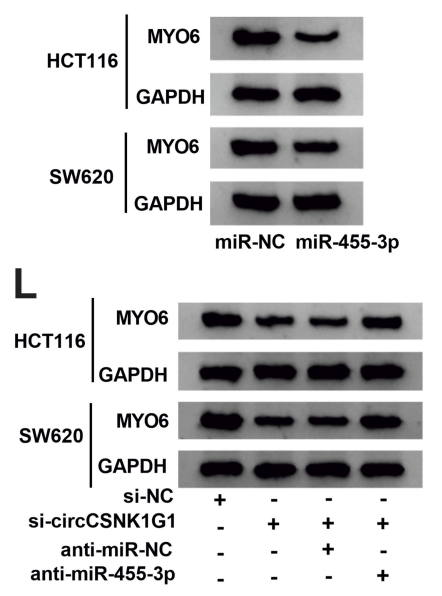

E

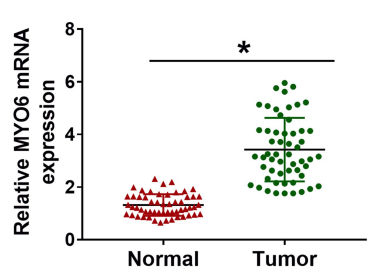

H
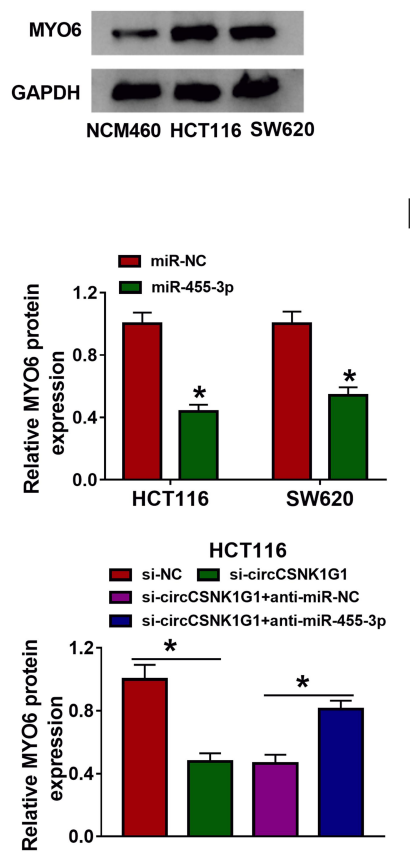

MYO6, a Target of miR-455-3p, Was

Regulated by circCSNKIGI via miR-455$3 p$

To further establish a "circRNA-miRNA-mRNA" network, the potential target mRNAs of miR-455-3p were identified. MiR-455-3p directly bound to MYO6 3'-UTR through several targeting sites, and the sites in MYO6 3'-

B

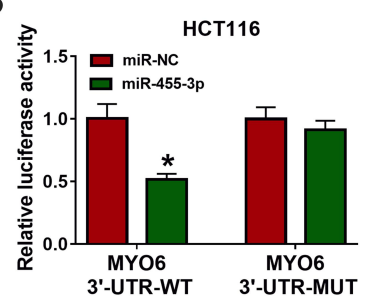

C

F
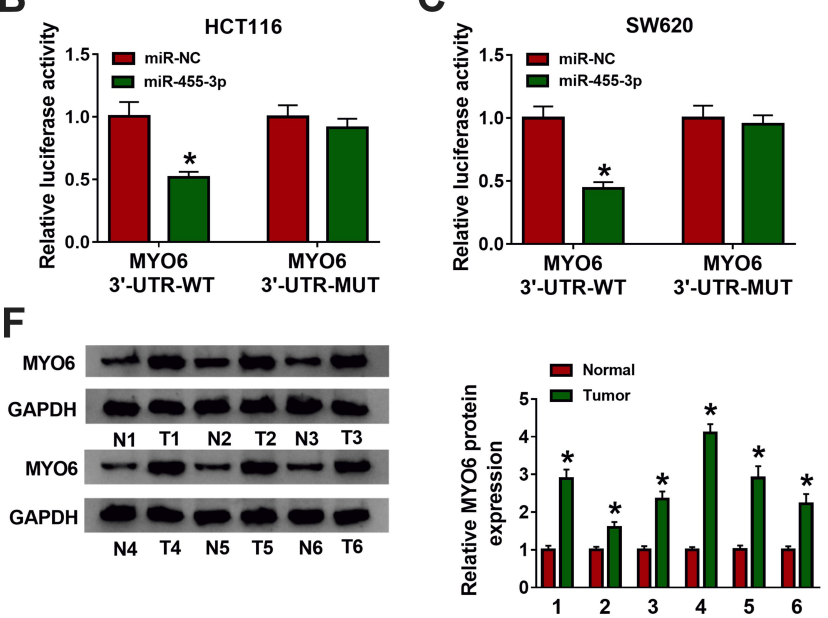

I
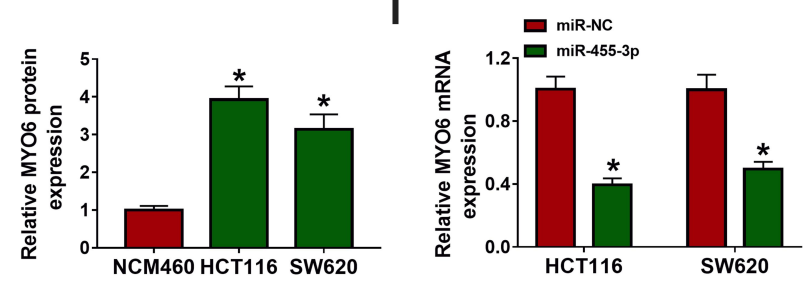

K
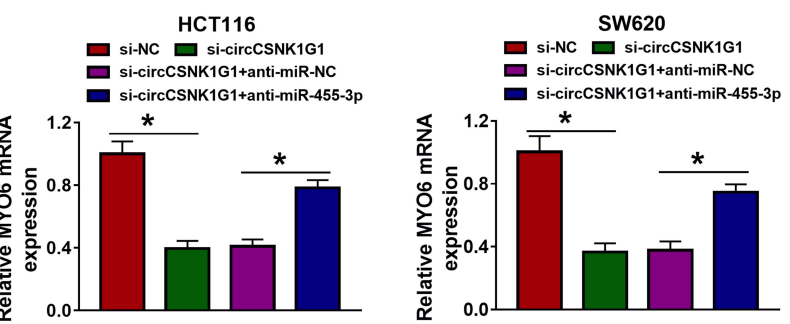

Figure 5 MYO6 was a target of miR-455-3p. (A) Using the bioinformatics tool starBase to predict the interaction between MYO6 and miR-455-3p. (B and C) Using dualluciferase reporter assay to verify the interaction between MYO6 and miR-455-3p. (D) The expression of MYO6 in COAD tissues was acquired from the TCGA data. (E and F) The expression of MYO6 in clinical tissues was detected by qRT-PCR and Western blot. ( $\mathbf{G}$ and $\mathbf{H})$ The expression of MYO6 in cell lines was detected by qRT-PCR and Western blot. ( $\mathbf{I}$ and $\mathbf{J}$ ) The expression of MYO6 in HCTI 16 and SW620 cells transfected with miR-455-3p or miR-NC was detected by qRT-PCR and Western blot. (K and L) The expression of MYO6 in HCTII6 and SW620 cells transfected with si-circCSNKIGI, si-NC, si-circCSNKIGI+anti-miR-455-3p or si-circCSNKIGI+anti-miR-NC was detected by $\mathrm{qRT}$-PCR and Western blot. $* P<0.05$. 
UTR were mutated to conduct dual-luciferase reporter assay (Figure 5A). The luciferase activities in HCT116 and SW620 cells cotransfected with MYO6 3'-UTR-WT and miR-455-3p was remarkably declined, while cotransfection of MYO6 3'-UTR-MUT and miR-455-3p hardly changed the luciferase activities (Figure 5B and C), suggesting that MYO6 was a target of miR-455-3p. Besides, MYO6 was observed to be upregulated in colon adenocarcinoma (COAD) tissues $(n=275)$ compared with that in normal tissues $(\mathrm{n}=349)$ from the TCGA data (Figure 5D). The data of MYO6 expression in our clinical tumor tissues $(n=55)$ were also enhanced compared to that in normal tissues ( $\mathrm{n}=55$ ) (Figure 5E and F). Similarly, the expression of MYO6 was elevated in HCT116 and SW620 cells compared with that in NCM460 cells (Figure 5G and H). Moreover, MYO6 was significantly downregulated in HCT116 and SW620 cells transfected with miR-455-3p compared to miR-NC (Figure 5I and J). More importantly, the expression of MYO6 in cells transfected with sicircCSNK1G1 was markedly decreased, while in cells transfected with si-circCSNK1G1+anti-miR-455-3p, the expression of MYO6 was largely promoted (Figure 5K and L). Briefly, circCSNK1G1 positively regulated MYO6 expression by targeting miR-455-3p.

\section{MiR-455-3p Restoration Weakened} Proliferation, Migration and Invasion and Induced Apoptosis and Cell Cycle Arrest in HCTII 6 and SW620 Cells by

\section{Degrading MYO6}

The expression of MYO6 impaired in HCT116 and SW620 cells transfected with miR-455-3p was substantially recovered in cells transfected with miR-455-3p +MYO6 (Figure 6A). Single miR-455-3p restoration prominently diminished the OD value and the number of colonies, while combined miR-455-3p restoration and MYO6 overexpression heightened the OD value and the number of colonies (Figure 6B-D). Besides, single miR455-3p restoration notably promoted the number of apoptotic cells, while combined miR-455-3p restoration and MYO6 overexpression lessened the number of apoptotic cells (Figure 6E). Meanwhile, the expression of Bax and c-caspase-3 was increased in cells transfected with miR455-3p but partly declined in cells transfected with miR455-3p+MYO6, while the expression of Bcl-2 was opposite to them (Figure 6F and G). Moreover, cell cycle arrest induced by miR-455-3p restoration was attenuated by the reintroduction of MYO6 (Figure 6H and I). The capacities of cell migration and cell invasion were blocked in cells transfected with miR-455-3p but reinforced in cells transfected with miR-455-3p+MYO6 (Figure 6J and K). These data suggested that miR-455-3p blocked the development of CRC by suppressing MYO6.

\section{CircCSNKIGI Knockdown Blocked Tumor Growth in vivo via the miR-455- 3p/MYO6 Axis}

Further experiments were performed to explore the role of circCSNK1G1 in vivo. SW620 cells infected with shcircCSNK1G1 or sh-NC were implanted into the subcutaneous part of nude mice. According to the data, circCSNK1G1 knockdown strikingly lessened tumor volume and tumor weight (Figure 7A and B). Tumor tissues were removed for expression analysis. The expression of circCSNK1G1 in tissues from the shcircCSNK1G1 group was pronouncedly decreased compared with that from the sh-NC group (Figure 7C), while the expression of miR-455-3p was increased in tissues from the sh-circCSNK1G1 group (Figure 7D). The expression of MYO6 was strikingly declined in tissues from the sh-circCSNK1G1 group at both mRNA and protein levels (Figure 7E and F). The data indicated that circCSNK1G1 knockdown inhibited tumor growth in vivo by the miR455-3p/MYO6 axis.

\section{Discussion}

Reliable biomarkers play an important role in the diagnosis, prognosis and prediction of CRC with great significance for the treatment of specific CRC patients. ${ }^{27}$ The development and research of ncRNAs expand our understanding of their biological contribution in the pathogenesis of cancers, and also provide important insights into their feasibility as clinical biomarkers for cancer. ${ }^{27,28}$ Here, we identified a differently expressed circRNA (circCSNK1G1) in CRC tissues and non-tumor tissues from the GEO database. We harbored the ideas that circCSNK1G1 was a novel CRC-related oncogene because circCSNK1G1 knockdown blocked CRC cell proliferation, migration and invasion, and tumor growth in vivo. Further, circCSNK1G1 indirectly mediated the expression of miR455-3p-targeted MYO6, and the circCSNK1G1/miR-4553p/MYO6 network was an underlying mechanism of circCSNK1G1 function in CRC. 

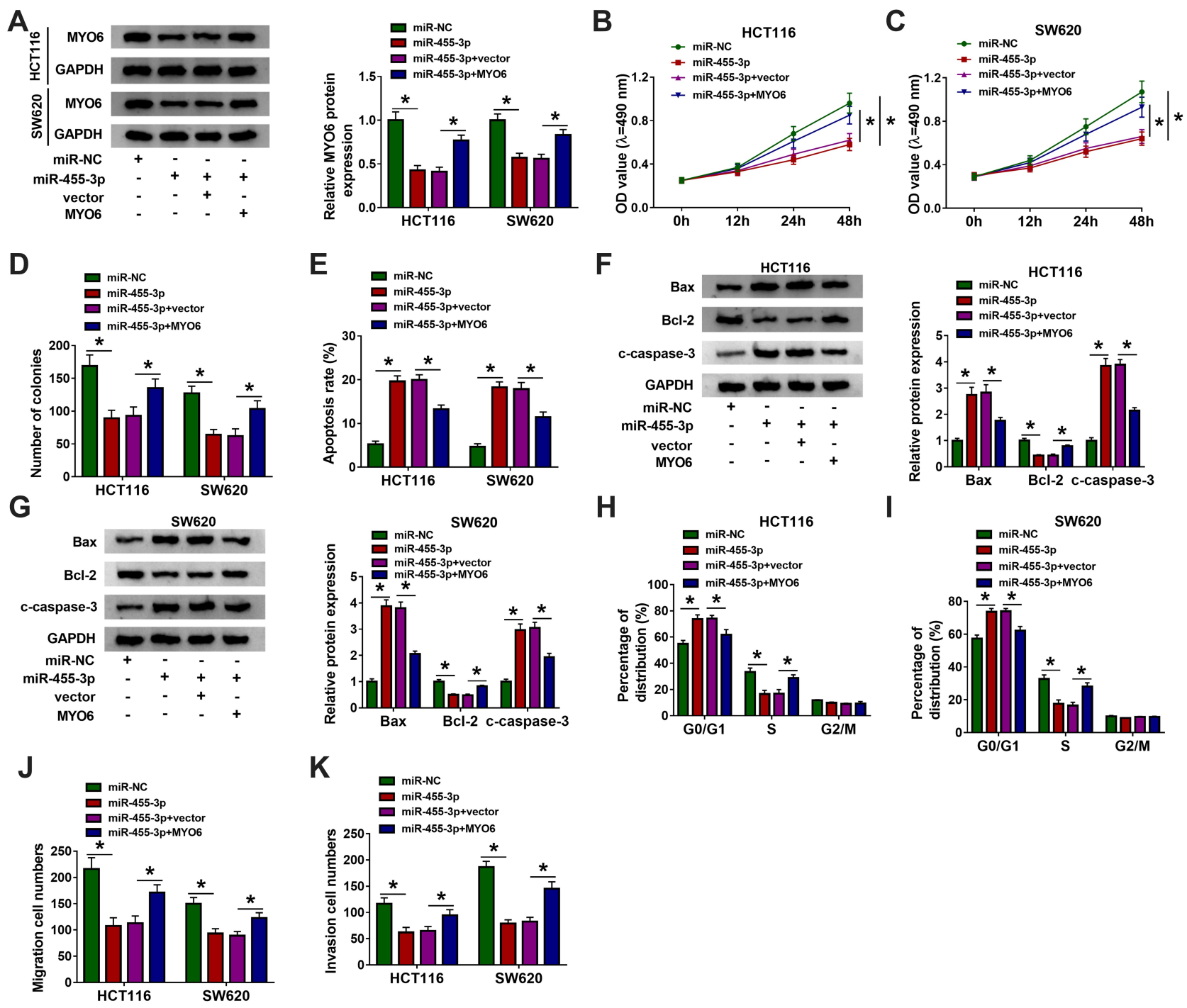

Figure 6 MiR-455-3p restoration blocked CRC development in vitro by targeting MYO6. HCTII 6 and SW620 cells were introduced with miR-455-3p, miR-NC, miR-4553p+MYO6 or miR-455-3p+vector. (A) The expression of MYO6 in these transfected cells was quantified by Western blot. (B-D) Cell proliferation was evaluated by MTT assay and colony formation assay in indicated cells. (E) Cell apoptosis was investigated by flow cytometry assay in indicated cells. (F and $\mathbf{G})$ The expression of Bax, Bcl-2 and c-caspase-3 in indicated cells was quantified by Western blot. ( $\mathbf{H}$ and $\mathbf{I})$ Cell cycle distribution in indicated cells was monitored using flow cytometry assay. ( $\mathbf{J}$ and $\mathbf{K})$ Cell migration and invasion in indicated cells were determined using transwell assay. $* P<0.05$.

Chen et al performed the microarray of circRNA profile using $10 \mathrm{CRC}$ tissues and matched non-tumor tissues, and circCSNK1G1 was one of the significantly upregulated circRNAs in CRC tissues. ${ }^{16}$ Consistent with this result, the expression of circCSNK1G1 in our clinical CRC tissues was also elevated compared to adjacent normal tissues. Moreover, circCSNK1G1 (circ_0001955) was documented to accelerate the tumorigenesis of hepatocellular carcinoma. ${ }^{29}$ Circ_0001955 as a differently regulated molecule in hepatocellular carcinoma tissues and corresponding normal tissues was identified from GSE7852, GSE94508, and GSE97322 datasets. ${ }^{29}$ Downregulated of circ_0001955 weakened HCC cell colony formation and
HCC transplanted tumor growth, ${ }^{29}$ suggesting that circCSNK1G1 was an oncogene in HCC. In agreement with this conclusion, our data presented that silencing circCSNK1G1 in CRC cells impaired cell proliferation, migration and invasion but promoted cell apoptosis and cell cycle arrest. In addition, circCSNK1G1 knockdown also repressed tumor development in nude mice, hinting that circCSNK1G1 played similar carcinogenic effects in CRC.

Mechanically, the circRNA-miRNA-mRNA pathway was frequently highlighted in the mechanisms of cancer progression. ${ }^{29,30}$ We thus explored the underlying action mode of circCSNK1G1 in this manner. MiR-455-3p was 


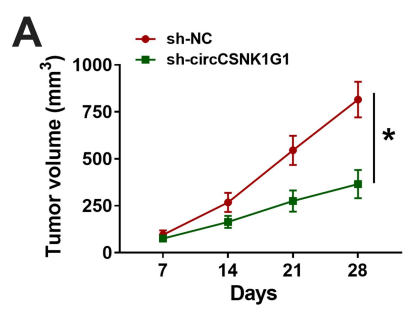

E

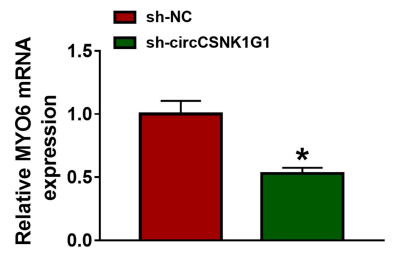

B

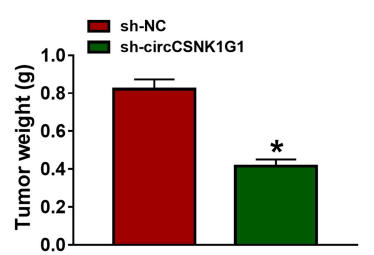

$\mathbf{F}$

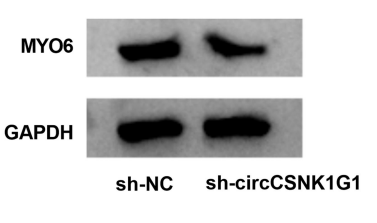

C
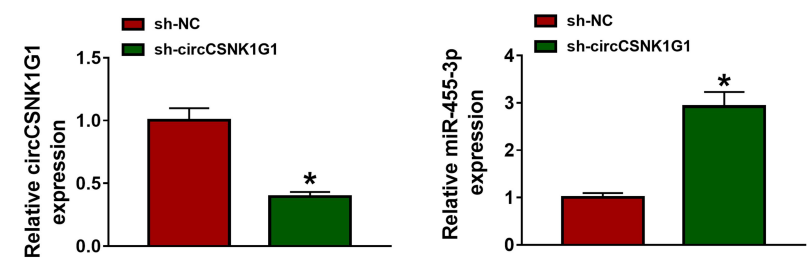

Figure 7 CircCSNKIGI knockdown suppressed tumor growth in vivo via the miR-455-3p/MYO6 axis. (A and B) After injection, tumor volume was measured once a week, and tumor weight was measured after 28 days. (C-E) The expression of circCSNKIGI, miR-455-3p and MYO6 in these removed tissues was detected by qRT-PCR. (F) The expression of MYO6 in the removed tissues was also detected by Western blot. $* P<0.05$.

predicted and validated to be a target of circCSNK1G1. Previous studies declaimed that miR-455-3p was a wide tumor suppressor and depleted cell proliferation, viability, invasion and migration in various cancers, including breast cancer, lung cancer and esophageal squamous cell carcinoma. ${ }^{31-33}$ In CRC, similarly, miR-455-3p expression was notably decreased in tumor tissues, and miR-455-3p mimics significantly blocked the malignant development of CRC. ${ }^{21,34}$ Consistently, our present data manifested that miR-455-3p inhibition reversed the effects of circCSNK1G1 downregulation, and miR-455-3p restoration played a similar role as circCSNK1G1 downregulation to depress CRC cell proliferation and migration.

Additionally, miR-455-3p directly bound to MYO6 3'UTR to degrade the expression and function of MYO6. Besides, MYO6 could be indirectly regulated by circCSNK1G1 through miR-455-3p. A previous study recorded that MYO6 was upregulated in CRC tissues, deletion of MYO6 blocked CRC cell proliferation and arrested cell cycle. ${ }^{24}$ MYO6 was also involved in CRC aggravation by acting as a target of miR-145 or miR-143 and was positively regulated by oncogenes (lncRNA SOX2-AS1 or IncRNA UCA1), thus promoting the development of CRC. ${ }^{35,36}$ In our views, we discovered that MYO6 was aberrantly enriched in CRC tissues and cells. MYO6 overexpression reversed the effects of miR-455-3p restoration, leading to aggressive cell proliferation and migration. In brief, MYO6 was an undisputed oncogene in CRC.

In summary, we held forceful evidence that circCSNK1G1 contributed to the tumorigenesis and progression of CRC by upregulating MYO6 via sponging miR-455-3p. Our data suggested that circCSNK1G1 was an ideal biomarker in CRC, and the circCSNK1G1/miR455-3p/MYO6 axis might be a promising strategy for CRC treatment.

\section{Funding}

This work was supported by the National Natural Science Foundation of China (81460372); Jiangxi Provincial Project for Distinguished Young Scholars grant (20162BCB23060).

\section{Disclosure}

The authors declare that they have no financial or nonfinancial conflicts of interest.

\section{References}

1. Marmol I, Sanchez-de-Diego C, Pradilla Dieste A, Cerrada E, Rodriguez Yoldi MJ. Colorectal carcinoma: a general overview and future perspectives in colorectal cancer. Int J Mol Sci. 2017;18(1):197. doi:10.3390/ijms 18010197

2. Torre LA, Bray F, Siegel RL, Ferlay J, Lortet-Tieulent J, Jemal A. Global cancer statistics, 2012. CA Cancer J Clin. 2015;65(2):87-108. doi:10.3322/caac. 21262

3. Testa U, Pelosi E, Castelli G. Colorectal cancer: genetic abnormalities, tumor progression, tumor heterogeneity, clonal evolution and tumor-initiating cells. Med Sci (Basel). 2018;6(2). doi:10.3390/ medsci6020031

4. Levin B, Lieberman DA, McFarland B, et al. Screening and surveillance for the early detection of colorectal cancer and adenomatous polyps, 2008: a joint guideline from the American Cancer Society, the US Multi-Society Task Force on Colorectal Cancer, and the American College of Radiology. CA Cancer J Clin. 2008;58(3):130-160. doi:10.3322/CA.2007.0018 
5. Eaden JA, Abrams KR, Mayberry JF. The risk of colorectal cancer in ulcerative colitis: a meta-analysis. Gut. 2001;48(4):526-535. doi:10.1136/gut.48.4.526

6. Johns LE, Houlston RS. A systematic review and meta-analysis of familial colorectal cancer risk. Am J Gastroenterol. 2001;96 (10):2992-3003. doi:10.1111/j.1572-0241.2001.04677.x

7. Mattick JS, Makunin IV. Non-coding RNA. Hum Mol Genet. 2006;15 Spec No 1:R17-29. doi:10.1093/hmg/ddl046

8. Vo JN, Cieslik M, Zhang Y, et al. The landscape of circular RNA in cancer. Cell. 2019;176(4):869-881 e813. doi:10.1016/j.cell.2018.12.021

9. Chen LL, Yang L. Regulation of circRNA biogenesis. RNA Biol. 2015;12(4):381-388. doi:10.1080/15476286.2015.1020271

10. Li Y, Zheng Q, Bao C, et al. Circular RNA is enriched and stable in exosomes: a promising biomarker for cancer diagnosis. Cell Res. 2015;25(8):981-984. doi:10.1038/cr.2015.82

11. Zhang Z, Yang T, Xiao J. Circular RNAs: promising biomarkers for human diseases. EBioMedicine. 2018;34:267-274. doi:10.1016/j. ebiom.2018.07.036

12. Li R, Wu B, Xia J, Ye L, Circular YX. RNA hsa_circRNA 102958 promotes tumorigenesis of colorectal cancer via miR-585/CDC25B axis. Cancer Manag Res. 2019;11:6887-6893. doi:10.2147/CMAR. S212180

13. Ng WL, Mohd Mohidin TB, Shukla K. Functional role of circular RNAs in cancer development and progression. RNA Biol. 2018;15 (8):995-1005. doi:10.1080/15476286.2018.1486659

14. Wang $\mathrm{X}$, Zhang $\mathrm{H}$, Yang $\mathrm{H}$, et al. Exosome-delivered circRNA promotes glycolysis to induce chemoresistance through the miR-122PKM2 axis in colorectal cancer. Mol Oncol. 2020;14(3):539-555. doi:10.1002/1878-0261.12629

15. Zaghlool A, Ameur A, Wu C, et al. Expression profiling and in situ screening of circular RNAs in human tissues. Sci Rep. 2018;8 (1):16953. doi:10.1038/s41598-018-35001-6

16. Chen Z, Ren R, Wan D, et al. Hsa_circ_101555 functions as a competing endogenous RNA of $\mathrm{miR}-5 \overline{9} 7-5 \mathrm{p}$ to promote colorectal cancer progression. Oncogene. 2019;38(32):6017-6034. doi:10.1038/ s41388-019-0857-8

17. To KK, Tong CW, Wu M, Cho WC. MicroRNAs in the prognosis and therapy of colorectal cancer: from bench to bedside. World $J$ Gastroenterol. 2018;24(27):2949-2973. doi:10.3748/wjg.v24. i27.2949

18. Cao L, Liu Y, Wang D, et al. MiR-760 suppresses human colorectal cancer growth by targeting BATF3/AP-1/cyclinD1 signaling. $J$ Exp Clin Cancer Res. 2018;37(1):83. doi:10.1186/s13046-018-0757-8

19. Zhang Z, Li J, Huang Y, et al. Upregulated miR-1258 regulates cell cycle and inhibits cell proliferation by directly targeting E2F8 in CRC. Cell Prolif. 2018;51(6):e12505. doi:10.1111/cpr.12505

20. Pichler M, Winter E, Stotz $M$, et al. Down-regulation of KRAS-interacting miRNA-143 predicts poor prognosis but not response to EGFR-targeted agents in colorectal cancer. $\mathrm{Br}$ $J$ Cancer. 2012;106(11):1826-1832. doi:10.1038/bjc.2012.175

21. Zheng J, Lin Z, Zhang L, Chen H. MicroRNA-455-3p inhibits tumor cell proliferation and induces apoptosis in HCT116 human colon cancer cells. Med Sci Monit. 2016;22:4431-4437. doi:10.12659/ msm.898452
22. Xu R, Fang XH, Zhong P. Myosin VI contributes to malignant proliferation of human glioma cells. Korean J Physiol Pharmacol. 2016;20(2):139-145. doi:10.4196/kjpp.2016.20.2.139

23. Buss F, Kendrick-Jones J. How are the cellular functions of myosin VI regulated within the cell? Biochem Biophys Res Commun. 2008;369(1):165-175. doi:10.1016/j.bbrc.2007.11.150

24. You W, Tan G, Sheng N, et al. Downregulation of myosin VI reduced cell growth and increased apoptosis in human colorectal cancer. Acta Biochim Biophys Sin (Shanghai). 2016;48(5):430-436. doi:10.1093/ abbs/gmw020

25. Livak KJ, Schmittgen TD. Analysis of relative gene expression data using real-time quantitative PCR and the 2(-Delta Delta $\mathrm{C}(\mathrm{T})$ ) Method. Methods. 2001;25(4):402-408. doi:10.1006/meth.2001.1262

26. Holdt LM, Kohlmaier A, Teupser D. Molecular roles and function of circular RNAs in eukaryotic cells. Cell Mol Life Sci. 2018;75 (6):1071-1098. doi:10.1007/s00018-017-2688-5

27. Okugawa Y, Grady WM, Goel A. Epigenetic alterations in colorectal cancer: emerging biomarkers. Gastroenterology. 2015;149(5):12041225 e1212. doi:10.1053/j.gastro.2015.07.011

28. Ling H, Fabbri M, Calin GA. MicroRNAs and other non-coding RNAs as targets for anticancer drug development. Nat Rev Drug Discov. 2013;12(11):847-865. doi:10.1038/nrd4140

29. Yao Z, Xu R, Yuan L, et al. Circ_0001955 facilitates hepatocellular carcinoma (HCC) tumorigenesis by sponging miR-516a-5p to release TRAF6 and MAPK11. Cell Death Dis. 2019;10(12):945. doi:10.1038/s41419-019-2176-y

30. Han B, Chao J, Yao H. Circular RNA and its mechanisms in disease: from the bench to the clinic. Pharmacol Ther. 2018;187:31-44. doi:10.1016/j.pharmthera.2018.01.010

31. Guo J, Liu C, Wang W, et al. Identification of serum miR-1915-3p and miR-455-3p as biomarkers for breast cancer. PLoS One. 2018;13 (7):e0200716. doi:10.1371/journal.pone.0200716

32. Gao X, Zhao H, Diao C, et al. miR-455-3p serves as prognostic factor and regulates the proliferation and migration of non-small cell lung cancer through targeting HOXB5. Biochem Biophys Res Commun. 2018;495(1):1074-1080. doi:10.1016/j.bbrc.2017.11.123

33. Liu A, Zhu J, Wu G, et al. Antagonizing miR-455-3p inhibits chemoresistance and aggressiveness in esophageal squamous cell carcinoma. Mol Cancer. 2017;16(1):106. doi:10.1186/s12943-0170669-9

34. Sun Y, Wang Y, Yang H, Xu Y, Yu H. miR-455-3p functions as a tumor suppressor in colorectal cancer and inhibits cell proliferation by targeting TPT1. Int J Clin Exp Pathol. 2018;11(5):2522-2529.

35. Wei AW, Li LF. Long non-coding RNA SOX21-AS1 sponges miR-145 to promote the tumorigenesis of colorectal cancer by targeting MYO6. Biomed Pharmacother. 2017;96:953-959. doi:10.1016/j. biopha.2017.11.145

36. Luan Y, Li X, Luan Y, et al. Circulating IncRNA UCA1 promotes malignancy of colorectal cancer via the miR-143/MYO6 axis. Mol Ther Nucleic Acids. 2020;19:790-803. doi:10.1016/j.omtn.2019.12.009
Cancer Management and Research

\section{Publish your work in this journal}

Cancer Management and Research is an international, peer-reviewed open access journal focusing on cancer research and the optimal use of preventative and integrated treatment interventions to achieve improved outcomes, enhanced survival and quality of life for the cancer patient.
The manuscript management system is completely online and includes a very quick and fair peer-review system, which is all easy to use. Visit http://www.dovepress.com/testimonials.php to read real quotes from published authors. 